\section{Guía para la preparación de un Módulo para el Aprendizaje Clínico Intensivo (MACl)}

Beltrán-Neira RJ, Ikeda-Artacho MC, Guerra C. Guía para la preparación de un Módulo para el Aprendizaje Clínico Intensivo (MACI). Rev Estomatol Herediana. 2011; 21(1):20-23.

RESUMEN

La enseñanza modular para el aprendizaje de la clínica intensiva ha probado ser un método de enseñanza excelente durante los últimos cuarenta años de actividades educativas en la Facultad de Estomatología de la Universidad Peruana CAYETANO HEREDIA, en Lima-Perú. Este artículo discute el proceso que se sigue para la planeación de la enseñanza modular. Cada función odontológica constituye un módulo de aprendizaje integral (Dominios: cognitivo, afectivo, psicomotor y volitivo). Aspectos importantes de la metodología son: enseñanza por equipo de profesores, participación activa del estudiante, práctica mínima en material inerte, demostración clínica y práctica inmediata en pacientes. La duración promedio de cada módulo es de doce horas en un plan continuo de actividades.

Palabras clave: EDUCACIÓN EN ODONTOLOGÍA / APRENDIZAJE CLÍNICO / ENSEÑANZA MODULAR.

How to prepare a module for dental clinical intensive learning (MACI. Sp.)

ABSTRACT

Modular teaching for intensive dental clinical learning has proved to be an excellent teaching model during the last forty years educating dental students at the Facultad de Estomatología of the Peruvian University CAYETANO HEREDIA, in Lima-Perú. This paper discusses the process to be followed for modular clinical teaching planning. Each integral module (Cognitive, affective, psychomotor and volitive domains), contains a dental function. Important features of this methodology are: teaching by teams of instructors, student active participation, minimum inert material practice, clinical demonstration by the instructors in patients, and immediate student clinical intervention in patients. The average duration for each module is of about twelve hours in a continuous plan of activities.

Key words: DENTAL EDUCATION / CLINICAL LEARNING / MODULAR TEACHING.
Roberto J. Beltrán Neira ${ }^{1}$ María Cristina Ikeda Artacho² Claudio Guerra ${ }^{3}$

'Profesor emérito. Profesor extraordinario investigador. Facultad de Estomatología. Universidad Peruana Cayetano Heredia.

2Docente del Departamento Académico de Clínica Estomatológica. Facultad de Estomatología.

Universidad Peruana Cayetano Heredia.

${ }^{3}$ Profesor de la Facultad de Odontología de la 3Profesor de la Facultad de Odontología de la
Universidad EI Salvador. Buenos Aires, Argentina.

\section{Correspondencia}

Roberto J. Beltrán Neira

La conquista 345, Lima 33 - Perú

Teléfono: 4368050

e-mail: robertobeltran@yahoo.com

Recibido : 17 de febrero de 2011

Aceptado : 15 de marzo de 2011
Una de las grandes creaciones estratégicas de la Facultad de Estomatología de la Universidad Peruana Cayetano Heredia (UPCH) en materia curricular fue y es -pues mantiene su vigencia- la modalidad de aprendizaje intensivo para el dominio de las funciones clínicas odontológicas. Es intención de esta comunicación aportar un camino que facilite a los profesores el diseño de módulos bajo esta modalidad, con la finalidad de lograr aprendizajes clínicos significativos en los educandos.

Se entiende por Función Clínica Odontológica a un elemento de Competencia Específica de la Estomatología, definido a partir de la Matriz Taxonómica de Competencias desarrollada por la Facultad de Estomatología de la UPCH. (Existe consenso a nivel mundial en que cada institución educativa debe definir para sus carreras las compe- tencias que el conocimiento de su ejercicio haga necesarias).

Desde la primera promoción de alumnos en 1972, el aprendizaje clínico se organizó en la UPCH modularmente bajo el concepto de "una función = un módulo".

\section{El Módulo para el Aprendizaje} Clínico Intensivo (MACI) se define como una construcción pedagógica diseñada para lograr la adquisición de los dominios cognitivo, afectivo, psicomotor $y$ volitivo que capaciten al alumno para la ejecución de una determinada función clínica odontológica en un tiempo mínimo. Mientras que en la clínica intensiva el aprendizaje es por funciones, en la clínica integral las funciones se integran en competencias para alcanzar el tratamiento integral del paciente.
La actual vigencia del concepto de aprendizaje por competencias enriquece conceptualmente el aprendizaje por funciones y contribuye a integrarlas en un nivel de mayor complejidad; esto ayuda al alumno a formarse una idea cabal del todo, uno de cuyos elementos es la función materia del módulo respectivo.

Este modelo de aprendizaje clínico odontológico intensivo se practicó con comodidad desde su implementación con promociones de hasta cuarenta alumnos, divididos en cuatro grupos de diez, que actúan en paralelo; cada grupo cuenta con la presencia de un instructor que ejerce de facilitador. Cuando el número de alumnos es superior, se requiere previamente la división de la promoción en dos grandes grupos y la conformación de un mayor número de subgrupos de diez, puesto que 
la instrucción es siempre grupal con participación activa del alumno.

Se debe considerar que el Aprendizaje Clínico Intensivo se sostiene en los aprendizajes previos logrados en las ciencias básicas -como anatomía, fisiología, farmacología, embriología e histología-, así como lo aprendido en las asignaturas de diagnóstico, conocimientos a los cuales habrá que remitir al grupo de estudiantes para su revisión a fin de lograr una apropiada integración básico-clínica.

GUÍA PARA LA PREPARACIÓN DE UN MÓDULO PARA EL APRENDIZAJE CLÍNICO INTENSIVO (MACI).-

\section{IDENTIFICACIÓN DE LA} FUNCIÓN que habrá de ser objeto del módulo.

Ello se facilita mediante la ubicación de la función (llamada también ELEMENTO DE COMPETENCIA) como integrante de una competencia, en la Matriz Taxonómica de Competencias Profesionales Odontológicas. Por ejemplo, el dominio de la función "Exodoncia no compleja", integrante de la competencia "Tratamiento de las afecciones bucales de índole quirúrgico", que a su vez es parte integrante del perfil profesional.

Cabe señalar que llamamos función a aquella "actividad que puede ser ejecutada independientemente y que es suficiente para solucionar una necesidad específica" por ejemplo, extracción de una tercera molar por necesidades ortodóncicas. Nos referimos a una actividad clínica asistencial que forma parte de una competencia; su objetivo no es solo que el alumno demuestre sus conocimientos sobre el tema, sino sus destrezas y, lo que es más importante, sus actitudes.

\section{REDACCIÓN DE LA SUMILLA. \\ La sumilla -que es la descripción del módulo- consiste en el resu- men o síntesis del mismo. Expone las características principales del módulo y las generalidades de su contenido, teniendo como referen- te el sílabo correspondiente a la asignatura a la cual pertenece di- cho módulo.}

\section{LISTADO DE LOS CONTENI- DOS Y DE LAS ESTRATEGIAS DIDÁCTICAS A EMPLEARSE EN EL MÓDULO. \\ Detalle de los contenidos que im- partirá el módulo así como del tipo de estrategias metodológicas que se utilizarán para lograr su apren- dizaje. Este material se seleccio- na a partir del sílabo correspon- diente a la asignatura a la cual pertenece el módulo.}

\section{DETALLE DE LOS PRODUC- TOS ESPERADOS Y EVALUA- CIÓN EN EL MÓDULO.}

Al final del módulo los alumnos deben estar en condiciones de ejecutar la actividad o procedimiento aprendido en la clínica intensiva, en pacientes de la clínica integral del período académico correspondiente. En particular, la estructura curricular de la Facultad de Estomatología de la Universidad Peruana Cayetano Heredia contempla un periodo clínico intensivo seguido de un período clínico integral, esquema que se repite en cada uno de tres últimos años académicos de la carrera profesional. En este sentido, el módulo debe ser claro en señalar al alumno los productos esperados a lo largo y al término del módulo así como el sistema de evaluación a emplearse, según fuera el caso.

Las formas de evaluación cuantitativa y cualitativa deben ser planteadas desde el inicio, con base en las exigencias de la competencia, a fin de que los estudiantes cuenten con todo lo necesario para generar y reunir las evidencias que les permitirán demostrar más tarde el dominio de la función.

\section{PROGRAMACIÓN DE LAS ACTIVIDADES PRESENCIA- LES Y NO PRESENCIALES DEL MÓDULO.}

Tomar en cuenta que, dependiendo del módulo, suelen requerirse unas 12 horas presenciales por función, distribuidas a lo largo de una semana. El módulo debe especificar claramente las actividades presenciales y no presenciales establecidas para el logro de los aprendizajes.

\section{LISTADO DE REFERENCIAS BIBLIOGRÁFICAS.}

Son una selección de aquellas referencias pertinentes al módulo y que son tomadas a partir de aquellas que aparecen en el sílabo de la asignatura a la que corresponde dicho módulo. Proporciona al alumno una guía para la búsqueda de información sobre la función que corresponde al módulo, convirtiéndose en un mecanismo de autoaprendizaje para reforzar lo aprendido.

IMPORTANCIA DEL DESARROLLOY SECUENCIA DE LAS ESTRATEGIAS DIDÁCTICAS

Junto con el contenido, parte importante en el diseño del módulo es la identificación de las estrategias didácticas que faciliten la enseñanza-aprendizaje-evaluación de la función escogida. Cada función puede 
requerir estrategias distintas; su selección debe considerar los tiempos disponibles para el módulo, puesto que existen diversas formas de alcanzar los mismos objetivos de aprendizaje.

Al inicio del módulo, es menester que el instructor responsable del módulo realice la presentación del MACI a los alumnos. La misma sitúa a la función -correspondiente al módulo- en el marco de la taxonomía de competencias odontológicas propia de la institución educativa, con una clara explicación de la relación entre los conceptos: necesidad de salud-competencia-función.

\section{Recordar:}

Presentación del trinomio: necesidad-competencia-función, correspondiente al módulo.

Las estrategias metodológicas empiezan por la búsqueda personal de información por parte del alumno y la conformación de grupos de trabajo, en donde se realice una explicación y discusión grupal del material revisado y de la información encontrada. Este método está muy desarrollado en la metodología de Aprendizaje Basado en la Solución de Problemas (ABSP o, simplemente, ABP).

Es de destacar que el módulo utiliza como método el aprendizaje participativo, de modo que el instructor no da clases expositivas, sino que facilita las discusiones grupales mediante las cuales contribuye a la fijación de los conceptos, actitudes, destrezas y voluntad para la búsqueda de integración y excelencia en el aprendizaje. Para tal fin, conviene que las mesas de discusión grupal no excedan un total de 8 a 10 alumnos.
En cuanto a la evaluación, corresponde a cada una de las distintas etapas componentes del módulo; en consecuencia, después de cada etapa en el módulo se debe desarrollar una mesa de discusión y evaluación.

\section{Recordar:}

Búsqueda individual de información + Discusión grupal para la profundización y fijación de conceptos $\rightarrow$ Evaluación en cada etapa.

A la discusión grupal suele continuar la ejecución de la demostración clínica por el instructor 0 , en su defecto, el estudio de un video que muestra la función. Idealmente conviene reforzar la demostración clínica por el instructor con el visionado de material audiovisual.

Existen funciones en las que está indicada alguna actividad preclínica o de laboratorio. Para estos casos, es importante señalar que ésta debe ser breve, realizada bajo supervisión y estar seguida prontamente de la experiencia clínica. Este es el sentido de la denominada preclínica, definida como la actividad que antecede a la práctica clínica. De acuerdo a lo antes expuesto, recordar que toda actividad debe finalizar con una discusión grupal de la práctica realizada, seguida de la evaluación correspondiente.

A la preclínica sigue la primera ejecución clínica del alumno. Las condiciones de su realización incluyen que sea en paciente seleccionado y bajo supervisión docente con retroalimentación inmediata. Su objetivo se orienta al logro de la ejecución consciente; se entiende por ejecución consciente a aquella según la cual el ejecutante mantiene una vigilancia permanente de cada una de la tareas, pasos, movimientos y momentos críticos de una determinada función.

A continuación pueden requerirse ejecuciones clínicas complementarias. Las condiciones de su realización también incluyen pacientes seleccionados y bajo supervisión docente con reatroalimentación inmediata. Los alumnos deben completar la secuencia en el tiempo indicado. Es responsabilidad del instructor proporcionar al alumno las facilidades para completar a tiempo el aprendizaje de la función. El objetivo de las ejecuciones clínicas complementarias se orienta al refuerzo de la ejecución consciente con tendencia a la ejecución automatizada; se entiende por ejecución automatizada a aquella que no consumirá recursos de atención permitiendo realizar observaciones en paralelo, aunque el estado de alerta debe permanecer constante a lo largo de todo el procedimiento. La ejecución automatizada se va logrando progresivamente en las clínicas integrales.

A las ejecuciones clínicas sigue una etapa reflexiva, a través de una mesa redonda de recapitulación y fijación de conceptos fundamentales de todos los dominios: cognitivo, afectivo, psicomotor y volitivo, seguida de la discusión grupal sobre la práctica individual.

El módulo concluye con una evaluación cuali-cuantitativa. El reconocimiento mediante evaluación cualitativa de la competencia del alumno actúa como mecanismo para el control de la integración de los dominios cognitivo, afectivo, psicomotor y volitivo del aprendizaje. 
Finalmente, se recogen las opiniones de los cursantes sobre su experiencia en el módulo.

\section{Recordar:}

Demostración clínica a cargo del instructor o video de la función

$$
\downarrow
$$

Uso de simuladores (preclínica) en casos en que fuese conveniente o indispensable

Primera ejecución clínica del alumno

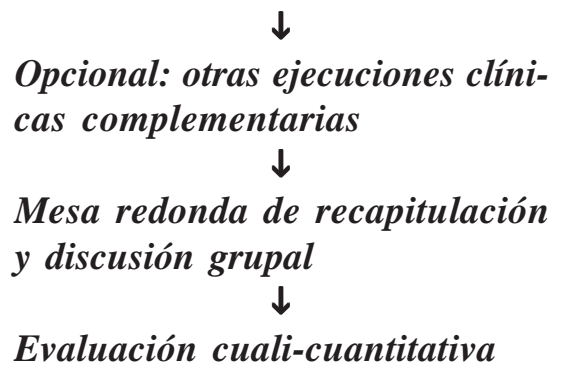

Para finalizar este artículo, consideramos importante dar a conocer a la comunidad académica que la Facultad de Estomatología de la Universidad Peruana Cayetano Heredia -hace más de cuarenta años- fue la primera en su campo en utilizar el concepto de enseñanza-aprendizaje por competencias, por entonces llamadas funciones, el cual en la actualidad figura entre las corrientes más importantes en educación.

\section{Referencias bibliográficas}

1. 1. Beltrán R. Modelo para el Diseño Curricular en Ciencias de la Salud. ALAFO Julio 1975.

2. Beltrán R, Bernal J. Aprendizaje por Funciones ALAFO Enero 1976.

3. Beltrán R, Aguayo H, Donayre F. Una Función: Un Módulo. ALAFO Enero 1979.

4. Beltrán R. Educación en Odontología. Manual del Profesor. 246 p. Facultad de Estomatología, Universidad Peruana Cayetano Heredia. 1998.

5. Beltrán R. Beltrán-Aguilar ED. Aprendizaje Intensivo a Dedicación Exclusiva. 160 p. Facultad de Estomatología, Universidad Peruana Cayetano Heredia.2002

6. Beltrán R. Ikeda MC. Taxonomía de competencias en educación odontológica. Rev Estomatol
Herediana. 2004; 14(1-2):107-11.

7. Consejo Nacional de Evaluación, Acreditación y Certificación de la Calidad de la Educación Superior Universitaria (CONEAU). Curso de Especialización en Evaluación de Competencias Profesionales - Guía del Participante. Segunda edición. Lima, 2010.

8. Consejo Nacional de Evaluación, Acreditación y Certificación de la Calidad de la Educación Superior Universitaria (CONEAU). Seminario Internacional "Certificación Profesional por Competencias". Lima, 9-10 abril 2010.

9. Ikeda MC, Beltrán R. Aprendizaje basado en competencias El caso de odontología. 146 p. Editorial Universidad Peruana Cayetano Heredia. Lima, 2006.

10. Consejo Nacional de Evaluación, Acreditación y Certificación de la Calidad de la Educación Superior Universitaria (CONEAU).Compendio Técnico Normativo: Criterios y estándares para la certificación profesional en el Perú. Lima, diciembre 2009. 